\title{
Normal-mode analysis of infrared and Raman spectra of poly(vinyl fluoride)
}

\author{
J.W. Hong, J.B. Lando and J.L. Koenig * \\ Department of Macromolecular Science, Case Westem Reserve University, 10900 Euclid Avenue, Cleveland, OH 44106-7202 (USA) \\ S.H. Chough and S. Krimm \\ Department of Physics \& Macromolecular Research Center, University of Michigan, Ann Arbor, MI 48109 (USA)
}

(Received 20th May 1991)

\begin{abstract}
Infrared and Raman spectra of samples of poly(vinyl fluoride) (PVF) have been recorded. The vibrational spectra have been analyzed by means of normal-mode calculations. A force field was derived by using 2-fluorobutane as a model compound. Crowder's force field for hydrofluorocarbons was employed as a starting point and subsequently refined in application to secondary fluorides. A planar zigzag, syndiotactic single-chain model of crystalline PVF was submitted to be analyzed by this scheme. A comparison of observed infrared and Raman bands with frequencies calculated for syndiotactic PVF shows that PVF produced by conventional free radical polymerization has an atactic structure, supporting the ${ }^{19} \mathrm{~F}$-NMR results and conclusions reached by Koenig and Boerio. Band assignments in terms of atactic structure are proposed.
\end{abstract}

Keywords: Infrared spectrometry; Raman spectrometry; 2-Fluorobutane; Normal-mode analysis; Poly(vinyl fluoride)

Several researchers have reported infrared and Raman spectra of poly(vinyl fluoride) (PVF), and tentative band assignments have been made. The infrared spectra of PVF were used by Koenig and Mannion [1] as a basis for analyzing enchainment, conformational and configurational defects in the material. The Raman spectrum subsequently obtained by Koenig and Boerio [2] led to the interpretation of the stereochemical configuration of the polymer. The polarized infrared spectrum of PVF has also been interpreted by Zerbi and Cortelli [3]. However, there are still many aspects of the vibrational spectrum that are not completely understood. The stereoregularity of PVF, in particular, has been the subject of controversy. Both the X-ray work of Natta et al. [4] and Koenig's Raman spectra [2] subsequently ob- tained have led to the conclusion that the polymer is atactic. In addition, the tacticity of PVF has been recently studied by ${ }^{19} \mathrm{~F}$ solution NMR, and has been shown to be atactic [5-7]. Zerbi and Cortelli [3], on the other hand, have suggested a syndiotactic structure for PVF.

In the general case, the straightforward nature of interpreting NMR data would favor the atactic structural assignment. However, in the case of PVF, like PVC, the differential solubility of alternative chain configurations may play an important role. It is suspected that highly syndiotactic PVF, like highly syndiotactic crystalline PVC [8], is marginally soluble even in good solvents. This would imply that NMR analysis of syndiotactic PVF chain segments, localized in insoluble crystallites, would suffer a negative bias due to spin- 
lattice saturation effects [9]. Although the solubility of PVF would be expected to be less affected by tacticity than that of PVC, it is desirable to resolve analytical uncertainties related to this potential solubility factor by a solid state technique capable of studying both the ordered and amorphous regions of the sample.

Distinctions between different local configurations of a regular polymer chain can be made on the basis of normal-mode analysis of the vibrational spectrum, since the infrared and Raman bands, particularly in the $1500-700 \mathrm{~cm}^{-1}$ region, are sensitive to those structures. However, to date, the reliability of normal-mode calculations for PVF has been limited by the lack of an available force field appropriate to the secondary fluorides. We are presently in a position to carry out normal-mode calculations for PVF by having available vibrational spectra of 2-fluoropropane and 2-fluorobutane as well as their vibrational assignments. In this paper, we therefore present further experimental studies and the results of normal-mode calculations on PVF. We have recorded the infrared and Raman spectra of PVF. The polarized infrared spectra of oriented, and the Raman spectra of non-oriented PVF samples, have been obtained. In the normal-mode calculations, valence force constants have been transferred from the low-molecular-weight model compound, 2-fluorobutane.

\section{EXPERIMENTAL}

Laboratory-generated samples of PVF obtained from DuPont were used. Infrared spectra were recorded on a Digilab Model FTS-60 FT-IR spectrometer. Oriented samples were obtained by stretching the film four times its original length. Polarized infrared spectra were obtained with the electric vector parallel and perpendicular to the stretching direction. Raman spectra were obtained with a Spec 1403 double monochromator equipped with holographic gratings. Spectra were excited by the $514.5-\mathrm{nm}$ line of a Coherent Radiation 52 Argon Ion Laser and $90^{\circ}$ scattering geometry was used.
NORMAL-MODE CALCULATIONS OF MODEL COMPOUNDS

\author{
Derivation of the force field for secondary fluo- \\ rides
}

Although secondary hydrocarbon chlorides, bromides, and iodides have been extensively investigated spectroscopically [10-16], only a few studics have reported on secondary fluorides [1719]. The infrared spectrum of 2-fluoropropane has been recorded by Griffiths et al. [19] in the vapor state and in $\mathrm{CS}_{2}$ solution, and a very approximate qualitative description of the vibrational modes has been made. The vibrational spectra were also obtained for 2-fluoropropane and 2-fluorobutane by Crowder et al. [17,18], and vibrational assignments of these two materials were made with the aid of normal coordinate calculations. However, force constants were not given, and the potential energy distribution was reported only for 2-fluoropropane. We approached the task of obtaining an accurate force field for secondary fluoride by the method described here. A set of force constants for primary fluorides was available $[20,21]$, which required only a few additional constants to make it suitable for secondary fluorides. A reliable valence force field has also been published for saturated hydrocarbons [22,23]. The force field constants in this potential function were transferred, where applicable, to 2-fluorobutane. These combined sets of force constants were used to fit the frequencies in the $200-1200 \mathrm{~cm}^{-1}$ region reported by Crowder [17], for the three conformations of 2-fluorobutane. The final set of force constants is given in Table 1.

\section{RESULTS AND DISCUSSION}

Figure 1 presents the 2-fluorobutane molecule with its internal coordinates and Fig. 2 shows the possible conformations and their nomenclature. The full roster of coordinates used in the normal-mode calculations are listed in Table 2 . In Table 3 are given the calculated frequencies and potential energy distributions for the three conformations of this molecule. A detailed compari- 
TABLE 1

Force constants for secondary fluorides

\begin{tabular}{|c|c|c|c|}
\hline No. & Constant $^{\mathrm{a}}$ & Environment & $\begin{array}{l}\text { Calculated } \\
\text { value }^{\text {b }}\end{array}$ \\
\hline 1 & MH & ME & 4.7522 \\
\hline 2 & $\mathrm{CH}$ & HX & 4.902 \\
\hline 3 & $\mathrm{CH}$ & $\mathbf{H H}$ & 4.487 \\
\hline 4 & MC & MEHH & 4.4858 \\
\hline 5 & MC & MEHX & 4.413 \\
\hline 6 & $\mathrm{CC}$ & HXHH & 4.413 \\
\hline 7 & $\mathrm{CX}$ & HX & 5.100 \\
\hline 8 & $\mathrm{HCH}$ & HH & 0.5084 \\
\hline 9 & HMH & ME & 0.5397 \\
\hline 10 & $\mathrm{CMH}$ & HXME & 0.600 \\
\hline 11 & CMH & HHME & 0.6172 \\
\hline 12 & $\mathrm{MCH}$ & MEHX & 0.680 \\
\hline 13 & $\mathrm{MCH}$ & MEHH & 0.7041 \\
\hline 14 & $\mathrm{CCH}$ & HXHH & 0.610 \\
\hline 15 & MCX & MEHX & 1.262 \\
\hline 16 & $\mathrm{CCX}$ & HHHX & 1.162 \\
\hline 17 & HCX & HX & 1.000 \\
\hline 18 & $\mathrm{MCC}$ & MEHXHH & 1.100 \\
\hline 19 & $\mathbf{M} \cdot \mathbf{C}$ & MEHX & 0.0 .5 \\
\hline 20 & $\mathbf{M} \cdot \mathbf{C}$ & МЕНH & 0.0784 \\
\hline 21 & $\mathrm{C} \cdot \mathrm{C}$ & HXHH & 0.05 \\
\hline 22 & $\mathbf{M H}, \mathbf{M H}$ & ME & 0.001 \\
\hline 23 & $\mathrm{CH}, \mathrm{CH}$ & $\mathbf{H H}$ & 0.0167 \\
\hline 24 & $\mathrm{MC}, \mathrm{CC}$ & MEHXHH & 0.1480 \\
\hline 25 & $\mathrm{MC}, \mathrm{CX}$ & MEHX & 0.740 \\
\hline 26 & $\mathrm{CC}, \mathrm{CX}$ & HHHX & 0.840 \\
\hline 27 & $\mathrm{CM}, \mathrm{CMH}$ & MEHX & 0.206 \\
\hline 28 & $\mathrm{CM}, \mathrm{CMH}$ & MEHH & 0.1845 \\
\hline 29 & $\mathbf{M C}, \mathbf{M C H}$ & MEHX & 0.206 \\
\hline 30 & $\mathrm{MC}, \mathrm{MCH}$ & MEHH & 0.3065 \\
\hline 31 & MC,MCX & MEHX & 0.567 \\
\hline 32 & $\mathrm{MC}, \mathrm{MCC}$ & МЕНХНН & 0.273 \\
\hline 33 & $\mathrm{CC}, \mathrm{CCH}$ & HHHX & 0.206 \\
\hline 34 & $\mathrm{CC}, \mathrm{CCX}$ & HHHX & 0.567 \\
\hline 35 & $\mathrm{CC}, \mathrm{CCM}$ & HHHXME & 0.274 \\
\hline 36 & $\mathrm{CX}, \mathrm{HCX}$ & HX & 0.200 \\
\hline 37 & CX,MCX & MEHX & 0.500 \\
\hline 38 & $\mathrm{CX}, \mathrm{CCX}$ & HHHX & 0.500 \\
\hline 39 & $\mathrm{CX}, \mathrm{MCC}$ & МЕНXHН & -0.05 \\
\hline 40 & $\mathrm{MC}, \mathrm{HCC}$ & МЕHНHX & 0.106 \\
\hline 41 & $\mathrm{CC}, \mathrm{HCM}$ & HHHXME & 0.006 \\
\hline 42 & $\mathrm{MC}, \mathrm{XCC}$ & MEHXHH & 0.567 \\
\hline 43 & $\mathrm{CC}, \mathrm{XCM}$ & HHHXME & 0.567 \\
\hline 44 & HMH,HMH & ME & 0.0091 \\
\hline 45 & HMH,CMH & MEHH & 0.0039 \\
\hline 46 & $\mathrm{CMH}, \mathrm{CMH}$ & MEHH & 0.010 \\
\hline 47 & $\mathrm{CMH}, \mathrm{CMH}$ & MEHX & -0.0237 \\
\hline 48 & $\mathrm{CCH}, \mathrm{CCH}$ & HXHH & 0.05 \\
\hline 49 & MCH,MCH & MEHH & 0.074 \\
\hline 50 & MCH,HCC & MEHXHH & 0.10 \\
\hline 51 & $\mathrm{MCH}, \mathrm{HCC}$ & MEHHHX & 0.050 \\
\hline 52 & MCH,MCX & MEHX & 0.10 \\
\hline
\end{tabular}

TABLE 1 (continued)

\begin{tabular}{lllc}
\hline No. & Constant & Environment & $\begin{array}{c}\text { Calculated } \\
\text { value }^{\text {b }}\end{array}$ \\
\hline 53 & MCX,XCC & MEHXHH & 0.1 \\
54 & MCC,MCH & MEHXHH & -0.05 \\
55 & MCC,CCH & MEHXHH & 0.05 \\
56 & M·C,C·C & MEHXHH & 0.08 \\
57 & HMC,MCHT & MEHH & 0.1037 \\
58 & HMC,MCHG & MEHX & 0.05 \\
59 & HMC,MCHG & MEHH & -0.046 \\
60 & HMC,MCXT & MEHX & 0.063 \\
61 & HMC,MCXG & MEHX & 0.055 \\
62 & CCM,CMHT & HHHXME & 0.207 \\
63 & CCM,CMHG & HHHXME & 0.056 \\
64 & MCC,CCHG & MEHXHH & 0.106 \\
65 & HCC,CCXT & HHHX & 0.063 \\
66 & HCC,CCXG & HHHX & 0.055 \\
67 & HCC,CCHG & HXHH & -0.05 \\
68 & MCC,CCMT & MEHXHHME & -0.036 \\
\hline
\end{tabular}

${ }^{a} M$ is the carbon atom of $\mathrm{CH}_{3}$ group, $X$ is the $\mathrm{F}$ atom. b Stretch and stretch,stretch force constants are in mdyn $/ \AA$, while stretch,bend and bend force constants are in mdyn. ${ }^{\mathrm{c}} \mathbf{T}$ and $\mathrm{G}$ : trans and gauche.

son of observed and calculated frequencies is difficult because of the presence of three isomers in the liquid. However, the availability of published data on both liquid and solid forms of 2-fluorobutane permits certain inferences to be made based on observations of the effect of decreasing temperature on the spectrum of the material. Table 4 lists observed frequencies for solid and liquid 2-fluorobutane with calculated frequencies of the three isomers $\left(S_{\mathrm{hh}}, S_{\mathrm{ch}}\right.$, and $\left.S_{\mathrm{hh}}\right)$ shown in Fig. 2. We have assigned those bands which decrease in intensity in the solid state to the $S_{\mathrm{ch}}$ and $S_{\mathrm{hh}^{\prime}}$ conformations. Since the frequencies calculated for the $S_{\text {hh }}$ conformation fit the observed solid state frequencies better than those calculated for $S_{\mathrm{ch}}$, the solid state spectrum has been assumed to be accounted for mainly by the $S_{\mathrm{hh}}$ conformation.

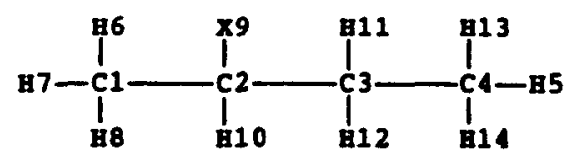

Fig. 1. 2-Fluorobutane with numbering corresponding to that used for internal coordinates ( $X=$ fluorine). 


\section{TABLE 2}

Internal coordinates for 2-fluorobutane

\begin{tabular}{|c|c|c|c|}
\hline \multicolumn{4}{|c|}{$\overline{\text { No. Bond }}{ }^{a}$} \\
\hline \multicolumn{4}{|c|}{ Stretching } \\
\hline & & & Bond length \\
\hline 1 & $(\mathrm{M}---\mathrm{C})$ & $(1-2)$ & 1.5400 \\
\hline 2 & $(\mathrm{M}---\mathrm{H})$ & $(1-6)$ & 1.0900 \\
\hline 3 & $(\mathrm{M}---\mathrm{H})$ & $(1-7)$ & 1.0900 \\
\hline 4 & $(\mathrm{M}--\mathrm{H})$ & $(1-8)$ & 1.0900 \\
\hline 5 & $(\mathrm{C}---\mathrm{C})$ & $(2-3)$ & 1.5400 \\
\hline 6 & $(\mathrm{C}---\mathrm{X})$ & $(2-9)$ & 1.3600 \\
\hline 7 & $(\mathrm{C}---\mathrm{H})$ & $(2-10)$ & 1.0900 \\
\hline 8 & $(\mathrm{C}---\mathrm{M})$ & $(3-4)$ & 1.5400 \\
\hline 9 & $(\mathrm{C}---\mathrm{H})$ & $(3-11)$ & 1.0900 \\
\hline 10 & $(\mathrm{C}---\mathrm{H})$ & $(3-12)$ & 1.0900 \\
\hline 11 & $(\mathrm{M}---\mathrm{H})$ & $(4-5)$ & 1.0900 \\
\hline 12 & $(\mathrm{M}---\mathrm{H})$ & $(4-13)$ & 1.0900 \\
\hline 13 & $(\mathrm{M}---\mathrm{H})$ & (4-14) & 1.0900 \\
\hline \multicolumn{4}{|c|}{ Bending } \\
\hline 14 & $(\mathbf{H}-\mathbf{M}-\mathrm{C})$ & $(6-1-2)$ & \\
\hline 15 & $(\mathrm{H}-\mathrm{M}-\mathrm{C})$ & $(7-1-2)$ & \\
\hline 16 & $(\mathbf{H}-\mathbf{M}-\mathrm{C})$ & $(8-1-2)$ & \\
\hline 17 & $(\mathbf{H}-\mathbf{M}-\mathrm{H})$ & $(6-1-7)$ & \\
\hline 18 & $(\mathbf{H}-\mathbf{M}-\mathbf{H})$ & $(6-1-8)$ & \\
\hline 19 & (H-M-H) & $(7-1-8)$ & \\
\hline 20 & $(\mathrm{M}-\mathrm{C}-\mathrm{C})$ & $(1-2-3)$ & \\
\hline 21 & $(\mathrm{M}-\mathrm{C}-\mathrm{X})$ & $(1-2-9)$ & \\
\hline 22 & $(\mathrm{M}-\mathrm{C}-\mathrm{H})$ & $(1-2-10)$ & \\
\hline 23 & $(\mathrm{X}-\mathrm{C}-\mathrm{C})$ & $(9-2-3)$ & \\
\hline 24 & $(\mathrm{X}-\mathrm{C}-\mathrm{H})$ & $(9-2-10)$ & \\
\hline 25 & $(\mathrm{H}-\mathrm{C}-\mathrm{C})$ & $(10-2-3)$ & \\
\hline 26 & (C-C-M) & $(2-3-4)$ & \\
\hline 27 & $(\mathrm{C}-\mathrm{C}-\mathrm{H})$ & $(2-3-11)$ & \\
\hline 28 & $(\mathrm{C}-\mathrm{C}-\mathrm{H})$ & $(2-3-12)$ & \\
\hline 29 & $(\mathrm{H}-\mathrm{C}-\mathrm{M})$ & $(11-3-4)$ & \\
\hline 30 & $(\mathrm{H}-\mathrm{C}-\mathrm{H})$ & $(11-3-12)$ & \\
\hline 31 & $(\mathrm{H}-\mathrm{C}-\mathrm{M})$ & $(12-3-4)$ & \\
\hline 32 & (C-M-H) & $(3-4-5)$ & \\
\hline 33 & $(\mathrm{C}-\mathrm{M}-\mathrm{H})$ & $(3-4-13)$ & \\
\hline 34 & $(\mathrm{C}-\mathrm{M}-\mathrm{H})$ & $(3-4-14)$ & \\
\hline 35 & $(\mathbf{H}-\mathbf{M}-\mathrm{H})$ & $(13-4-5)$ & \\
\hline 36 & (H-M-H) & (14-4-5) & \\
\hline 37 & $(\mathrm{H}-\mathrm{M}-\mathrm{H})$ & $(13-4-14)$ & \\
\hline
\end{tabular}

Torsions

\begin{tabular}{llllllllll}
38 & & & \multicolumn{4}{c}{ Connected atom indices } \\
39 & $(\mathrm{M} \cdot \mathrm{C})$ & $(1-2)$ & 6 & 7 & 8 & 3 & 9 & 10 \\
40 & $(\mathrm{C} \cdot \mathrm{M})$ & $(2-3)$ & 1 & 9 & 10 & 4 & 11 & 12 \\
& & $(3-4)$ & 2 & 11 & 12 & 5 & 13 & 14 \\
\hline
\end{tabular}

atomic weights: $\mathrm{M}=12.0111500 ; \mathrm{C}=12.0111500 ; \mathrm{H}=$ $1.0078250 ; \mathrm{X}=18.9984000 ; \mathrm{H}=1.0078250$.
TABLE 3

Calculated frequencies and potential energy distribution for 2-fluorobutane

\begin{tabular}{|c|c|}
\hline \multicolumn{2}{|l|}{ Band } \\
\hline \multicolumn{2}{|c|}{$S_{h h}$ conformation } \\
\hline 1448 & 77 (ME1 AB-2) 10(ME1 AB-1) \\
\hline 1414 & $\begin{array}{l}\text { 33(ME1 SBND) } 15(23 \mathrm{CC} \mathrm{S}) 12(\mathrm{HX} 2 \text { H-PD) } \\
\text { 11(1 } 2 \text { CC S) }\end{array}$ \\
\hline 1398 & 55(ME4 SBND) 18(HH3 WAG) \\
\hline 1381 & $\begin{array}{l}\text { 36(HH3 WAG) 36(ME4 SBND) 15(3 } 4 \text { CC S) } \\
\text { 12(ME1 SBND) }\end{array}$ \\
\hline 1276 & 37(HX2 H-PL) 32(ME1 SBND) \\
\hline 1220 & 41(HH3 TWS) 23(ME4 R-2) \\
\hline 1142 & $32(\mathrm{HX} 2 \mathrm{CX}-\mathrm{S}) 10(12 \mathrm{CC} \mathrm{S}) 10(\mathrm{HX} 2 \mathrm{DEF})$ \\
\hline 1084 & 18(ME1 R-2) 15(12 CC S) 14(34 CC S) \\
\hline 1028 & $\begin{array}{l}\text { 30(HX2 H-PL) } 13(12 \mathrm{CC} \mathrm{S}) 13(34 \mathrm{CC} \mathrm{S}) \\
\text { 11(HH3 WAG) }\end{array}$ \\
\hline 1010 & 36(ME1 R-1) 14(ME4 R-1) 11(34 CC S) \\
\hline 985 & 34(3 4 CC S) 24(ME4 R-1) 16(HH3 WAG) \\
\hline 971 & 32(HH3 TWS) 27(ME1 R-2) 19(ME4 R-2) \\
\hline 892 & $49(\mathrm{HX} 2 \mathrm{CX}-\mathrm{S})$ 26(ME1 R-2) 12(ME1 R-1) \\
\hline 820 & $\begin{array}{l}\text { 38(2 } 3 \text { CC S ) 16(ME4 R-1) 15(1 } 2 \text { CC S }) \\
\text { 14(ME1 R-1) }\end{array}$ \\
\hline 751 & 63(HH3 ROC) 43(ME4 R-2) \\
\hline 486 & 84(HX2 X-PD) \\
\hline 462 & 48(HX2 X-PL) 22(HH3 DEF) $14(12 \mathrm{CC} \mathrm{S})$ \\
\hline 348 & $\begin{array}{l}\text { 68(HX2 DEF) } 12(\mathrm{HX} 2 \mathrm{X}-\mathrm{PL}) 12(23 \mathrm{CC} \mathrm{S}) \\
\text { 10(HH3 DEF) }\end{array}$ \\
\hline 257 & 52(HH3 DEF) 20(HX2 DEF) 19(HX2 X-PL) \\
\hline \multicolumn{2}{|c|}{$S_{c h}$ conformation } \\
\hline 1448 & 85(ME4 AB-2) \\
\hline 1421 & $\begin{array}{l}\text { 24(ME1 SBND) 17(2 } 3 \mathrm{CC} \mathrm{S}) \text { 13(ME4 AB-1) } \\
\text { 11(HX2 H-PL) }\end{array}$ \\
\hline 1402 & 41(HH3 WAG) 19(ME1 SBND) 13(HX2 H-PD) \\
\hline 1391 & 90(ME4 SBND) 15(3 4 CC S) \\
\hline 1279 & 42(ME1 SBND) 32(HX2 H-PL) \\
\hline 1218 & 42(HH3 TWS) 25(ME4 R-2) \\
\hline 1150 & $\begin{array}{l}\text { 23(HX2 CX-S) } 12(12 \text { CC S) 12(ME1 R-1) } \\
\text { 10(HX2 DEF) }\end{array}$ \\
\hline 1095 & 28(ME1 R-2) 15(HX2 CX-S) 11(HX2 H-PL) \\
\hline 1042 & $43(34$ CC S) 17(ME4 R-1) \\
\hline 1015 & 31(HX2 H-PL) 11(3 4 CC S) 10(HH3 WAG) \\
\hline 975 & 26(ME4 R-1) 17(ME1 R-1) 11(ME1 R-2) \\
\hline 957 & $\begin{array}{l}\text { 26(HH3 TWS) 19(ME4 R-1) 14(1 } 2 \text { CC S) } \\
\text { 13(ME4 R-2) }\end{array}$ \\
\hline 903 & 43(HX2 CX-S) 35(ME1 R-2) 15(ME1 R-1) \\
\hline 802 & $36(23 \mathrm{CC} \mathrm{S}) 24(12 \mathrm{CC} \mathrm{S}) 10(\mathrm{ME} 1 \mathrm{R}-1)$ \\
\hline 750 & $57(\mathrm{HH} 3 \mathrm{ROC}) 39(\mathrm{ME} 4 \mathrm{R}-2)$ \\
\hline 482 & $80(\mathrm{HX} 2 \mathrm{X}-\mathrm{PD})$ \\
\hline 438 & 33(HX2 DEF) 32(HH3 DEF) 14(HX2 X-PD) \\
\hline 390 & 81(HX2 X-PL) \\
\hline 247 & 47(HX2 DEF) 47(HH3 DEF) 14(34 CCTO) \\
\hline
\end{tabular}


TABLE 3 (continued)

\begin{tabular}{|c|c|}
\hline \multicolumn{2}{|l|}{ Band } \\
\hline \multicolumn{2}{|c|}{$S_{h h^{\prime}}$ conformation } \\
\hline 1458 & 42(ME4 AB-1) 13(IIII3 BND) 10(2 $3 \mathrm{CC} \mathrm{S})$ \\
\hline 1452 & 71(HH3 BND) \\
\hline 1451 & 38(ME1 AB-2) 30(ME1 AB-1) \\
\hline 1449 & 50(ME1 AB-2) 27(ME1 AB-1) 12(ME4 AB-1) \\
\hline 1448 & 84(ME4 AB-2) \\
\hline 1433 & $\begin{array}{l}\text { 17(ME1 SBND) 16(ME1 AB-1) 12(ME4 AB-1) } \\
\text { 11(ME4 SBND) }\end{array}$ \\
\hline 1405 & $50(\mathrm{HH} 3 \mathrm{WAG}) 14(\mathrm{ME} 4 \mathrm{AB}-1)$ 13(3 4 CC S) \\
\hline 1385 & 71(ME4 SBND) 15(ME1 SBND) \\
\hline 1282 & 42(ME1 SBND) 32(HX2 H-PL) \\
\hline 1218 & 42(HH3 TWS) 25(ME4 R-2) \\
\hline 1152 & $\begin{array}{l}28(\mathrm{HX} 2 \mathrm{CX}-\mathrm{S}) 12(12 \mathrm{CC} \mathrm{S}) 11(\mathrm{HH} 3 \mathrm{ROC}) \\
10(\mathrm{HX} 2 \mathrm{DEF})\end{array}$ \\
\hline 1100 & 23(ME4 R-1) 19(3 4 CC S) 11(ME1 R-1) \\
\hline 1086 & 23(HX2 H-PL) 22(ME1 R-2) 13(12 CC S) \\
\hline 986 & $\begin{array}{l}\text { 32(ME4 R-1) 23(ME1 R-2) 15(HH3 WAG) } \\
\text { 10(HX2 H-PL) }\end{array}$ \\
\hline 967 & $\begin{array}{l}\text { 33(ME1 R-1) 22(HH3 TWS) } 18(34 \mathrm{CC} \mathrm{S}) \\
\text { 12(ME4 R-2) }\end{array}$ \\
\hline 933 & $\begin{array}{l}26(34 \text { CC S }) 19(12 \text { CC S) 18(HH3 TWS }) \\
\text { 11(ME4 R-2) }\end{array}$ \\
\hline 892 & 47(HX2 CX-S) 31(ME1 R-2) \\
\hline 813 & $41(23 \mathrm{CC} \mathrm{S}) 18(12 \mathrm{CC} \mathrm{S})$ \\
\hline 747 & 61(HH3 ROC) 41(ME4 R-2) \\
\hline 601 & 48(HX2 X-PD) 23(HH3 DEF) \\
\hline 422 & 73(HX2 X-PL) $10(23 \mathrm{CC} \mathrm{S})$ \\
\hline 366 & 80(HX2 DEF) \\
\hline 215 & 64(HH3 DEF) 36(HX2 X-PD) \\
\hline
\end{tabular}

From the potential energy distribution indicated in Table 3 , the most interesting parts of the spectra are the essentially delocalized frequencies of the C-F stretching modes. This result contrasts with other carbon-halogen stretching frequencies in primary and secondary alkyl halides. Crowder's results on primary and secondary fluo-

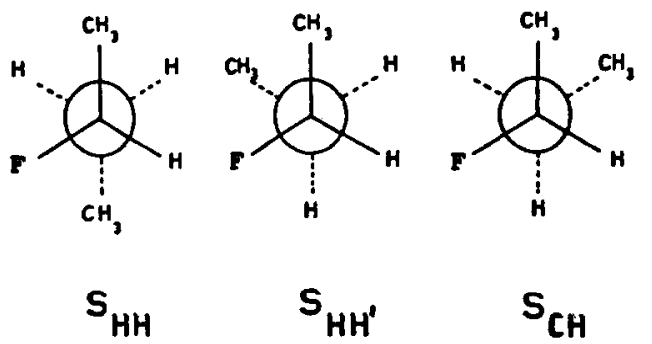

Fig. 2. Conformations of secondary fluorides.
TABLE 4

Assignments to observed frequencies of 2-fluorobutane

\begin{tabular}{|c|c|c|c|c|}
\hline \multicolumn{2}{|l|}{ Observed } & \multicolumn{3}{|c|}{ Calculated } \\
\hline Solid & Liquid & $\overline{S_{\mathrm{hh}}}$ & $S_{\mathrm{ch}}$ & $\overline{S_{\mathrm{hh}^{\prime}}}$ \\
\hline & $\begin{array}{l}1470 \mathrm{~m} \\
1458 \mathrm{~m}\end{array}$ & & & \\
\hline $1449 \mathrm{mw}$ & $1450 \mathrm{w}$ & & & 1448 \\
\hline $1386 \mathrm{mw}$ & $1387 \mathrm{~m}$ & 1398 & 1391 & 1405 \\
\hline $1378 \mathrm{w}$ & $1380 \mathrm{sh}$ & 1381 & & 1385 \\
\hline $1351 \mathrm{w}$ & $1350 \mathrm{sh}$ & & & \\
\hline $1341 \mathrm{w}$ & $1342 \mathrm{mw}$ & & & \\
\hline $1303 \mathrm{vw}$ & $1306 \mathrm{vw}$ & & & \\
\hline $1272 \mathrm{vw}$ & $1273 \mathrm{vw}$ & 1276 & 1279 & 1282 \\
\hline $1180 \mathrm{mw}$ & $1177 \mathrm{mw}$ & & & \\
\hline $1155 \mathrm{ww}$ & $1154 \mathrm{w}$ & & & \\
\hline $1128 \mathrm{mw}$ & $1133 \mathrm{~ms}$ & & & \\
\hline \multirow[t]{2}{*}{$1116 \mathrm{~ms}$} & $1120 \mathrm{~ms}$ & 1142 & 1150 & 1152 \\
\hline & $1103 \mathrm{~m}$ & & 1095 & 1100 \\
\hline $1033 \mathrm{~m}$ & $1033 \mathrm{~ms}$ & 1028 & 1042 & \\
\hline $998 \mathrm{mw}$ & $993 \mathrm{~m}$ & 1010 & 1015 & \\
\hline \multirow[t]{2}{*}{$984 \mathrm{w}$} & $985 \mathrm{~m}$ & 985 & 975 & 986 \\
\hline & $978 w$ & & & 967 \\
\hline $971 \mathrm{~m}$ & $970 \mathrm{~s}$ & 971 & 957 & \\
\hline 889 s & $895 \mathrm{~s}$ & 892 & 903 & 892 \\
\hline $824 \mathrm{~m}$ & $824 \mathrm{~m}$ & 820 & 802 & 813 \\
\hline \multirow[t]{2}{*}{$765 w$} & $765 \mathrm{w}$ & 751 & 750 & 747 \\
\hline & $595 \mathrm{vw}$ & & 601 & \\
\hline $495 w$ & $495 w$ & 486 & 482 & \\
\hline \multirow[t]{6}{*}{$462 w$} & $461 w$ & 462 & 438 & \\
\hline & $422 \mathrm{vw}$ & & 422 & \\
\hline & $383 w$ & & 390 & \\
\hline & $360 \mathrm{vw}$ & 348 & & 366 \\
\hline & $253 \mathrm{w}$ & 257 & 247 & \\
\hline & $228 \mathrm{vw}$ & & & 215 \\
\hline
\end{tabular}

rides showed the same phenomena. A study of Table 4 indicates that the fit between calculated and observed frequencies below $1200 \mathrm{~cm}^{-1}$ is quite satisfactory. On the other hand, the fit in the region above $1200 \mathrm{~cm}^{-1}$ grows increasingly poorer with increased wavenumber. Problems with the force field related to this region are present, but it can be said that the force constants represent the best derivable fit to the available data. In order to have a more reliable force field for secondary fluorides, both infrared and Raman data for 2-fluoropropane and 2-fluorobutane are required. The resulting force field should additionally be tested on other related model compounds, such as 3-fluoropentane and 2,4-difluoropentane. Therefore, although the 
present force field cannot be considered totally general in application to secondary fluorides, it represents a starting point on which to build, and should be capable of lending insight to the interpretation of the vibrational spectra of this class of compounds. In particular, we feel that its application to the analysis of the spectra of poly(vinyl fluoride) is justified.

\section{NORMAL-MODE CALCULATION OF PVF}

The normal-mode calculations were done on a single chain for the stereoregular and regioregular structures, i.e., no interchain forces are considered. Although the classical approach to the vibrational analysis of polymers in terms of an infinite chain mode with perfect chemical, stereoregular and conformational structures is not valid for real polymers, the vibrational analysis of a disordered material can be more easily performed if the vibrational spectrum of the infinite perfect model is well known and is used as a reference point in the comparison.

The structural parameters used were the same as for the secondary fluorides. The bond angles were assumed to be tetrahedral. Cartesian coordinates were calculated for the planar zigzag structures of the isolated syndiotactic PVF chain. The symmetry coordinates, constructed from the internal coordinates defined in the standard way (Table 5), are listed in Table 6 with numbcring of

\section{TABLE 5}

Internal coordinates for syndiotactic PVF

\begin{tabular}{|c|c|c|c|c|c|}
\hline \multicolumn{2}{|c|}{ Bond lengths } & \multicolumn{4}{|c|}{ Bond angles } \\
\hline$R^{\mathrm{I}}$ & $1-2$ & $\phi_{1}^{\mathrm{II}}$ & $6-1-2$ & $\theta^{I}$ & $1-2-9$ \\
\hline$R^{\mathrm{II}}$ & $2-3$ & $\phi_{2}^{1 I}$ & $1-2-3$ & $\Theta^{I I}$ & $1-2-10$ \\
\hline$R^{\mathrm{III}}$ & $3-4$ & $\phi_{3}^{\text {II }}$ & $2-3-4$ & $\Theta^{I I I}$ & $9-2-3$ \\
\hline$R^{\mathrm{IV}}$ & $4-5$ & $\phi_{4}^{\mathrm{II}}$ & $3-4-5$ & $\Theta^{\mathrm{IV}}$ & $10-2-3$ \\
\hline$r_{1}^{\mathrm{I}}$ & $1-7$ & $\phi_{1}^{I I I}$ & $7-1-8$ & $\theta^{\mathrm{I}}$ & $2-3-11$ \\
\hline$r_{2}^{1}$ & $1-8$ & $\phi_{2}^{\mathrm{III}}$ & $9-2-10$ & $\theta^{\mathrm{II}}$ & $2-3-12$ \\
\hline$r_{1}^{\mathrm{II}}$ & $2-9$ & $\phi_{3}^{\mathrm{III}}$ & $11-3-12$ & $\theta^{\mathrm{III}}$ & $4-3-11$ \\
\hline$r_{2}^{I I}$ & $2-10$ & $\phi_{4}^{\text {III }}$ & $13-4-14$ & $\theta^{\mathrm{IV}}$ & $4-3-12$ \\
\hline$r_{1}^{\text {IIII }}$ & $3-11$ & $\phi_{1}^{\text {IV V }}$ & $6-1-7$ & $\phi_{1}^{\mathrm{I}}$ & $3-4-13$ \\
\hline$r_{2}^{1 I I I}$ & $3-12$ & $\phi_{2}^{I V}$ & $6-1-8$ & $\phi \frac{1}{2}$ & $3-4-14$ \\
\hline$r_{1}^{\mathrm{IV} V}$ & $4-13$ & $\phi_{3}^{\mathrm{IV}}$ & $7-1-2$ & $\phi 3$ & $5-4-13$ \\
\hline$r_{2}^{\mathrm{IV}}$ & $4-14$ & $\phi_{4}^{\mathrm{IVV}}$ & $8-1-2$ & $\phi_{4}^{\mathrm{I}}$ & $5-4-14$ \\
\hline
\end{tabular}

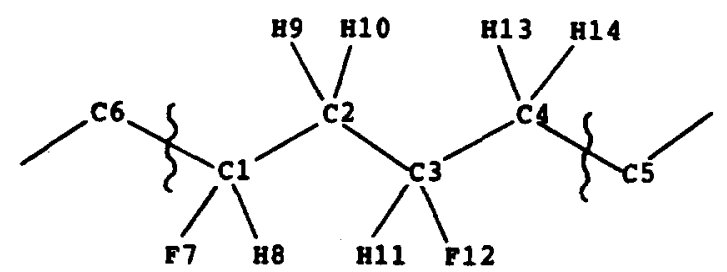

Fig. 3. Structural unit of syndiotactic PVF, with atom numbering.

atoms shown in Fig. 3. The force field for the single chain was the same as that used for secondary fluorides. The calculated frequencies are compared with the observed in Table 7 in which the assignments of the bands based on the calculated potential energy distribution are also given.

\section{RESULTS AND DISCUSSION}

For atactic poly(vinyl fluoride), all the spectrally active vibrational modes occur both in the infrared and Raman spectra. Planar syndiotactic PVF has a symmetry isomorphous with the factor group $C_{2 v}$ [4]. The normal modes for the planar syndiotactic structure are distributed among the species as Raman and infrared active $\left(A_{1}, B_{1}\right.$, $B_{2}$ ) modes and only Raman active $A_{2}$ modes [24]. PVF with a planar isotactic structure has a symmetric isomorphous with the factor group $C_{s}$. The normal modes for this isotactic structure may be divided among the symmetry species as $A^{\prime}$ (in phase) and $A^{\prime \prime}$ (out of phase). All vibrations are active in both the infrared and Raman spectra. The symmetry analysis indicates that only syndiotactic placements lead to differences in infrared and Raman selection rules in PVF.

Polarized infrared spectra of the oriented PVF film are shown in Fig. 4 and the Raman spectrum is shown in Fig. 5. The observed infrared and Raman frequencies are listed in Table 6 and compared with calculated frequencies of the syndiotactic structure. In this table we listed only modes in the $1500-700 \mathrm{~cm}^{-1}$ region. Analysis of Figs. 4 and 5 with Table 7 indicates that some Raman bands desire special consideration, among them are the bands at $1150 \mathrm{~cm}^{-1}$ and at 1436 
$\mathrm{cm}^{-1}$. As assigned by Koenig and Boerio [2], these bands may correspond to infrared bands at $1144 \mathrm{~cm}^{-1}$. However, the band at $1144 \mathrm{~cm}^{-1}$, which was assigned as $\nu(\mathrm{CF})$, should be weaker in the Raman than in the IR spectrum, which is the opposite of the observed.

TABLE 6

Symmetry coordinates for syndiotactic poly(vinyl fluoride) $\left(C_{2 v}\right.$ symmetry)

$$
\begin{aligned}
& a_{1} \text { coordinates } \\
& S_{1}=\frac{1}{2}\left(\Delta R^{\mathrm{I}}+\Delta R^{\mathrm{II}}+\Delta R^{\mathrm{III}}+\Delta R^{\mathrm{IV}}\right) \\
& S_{2}=\frac{1}{2}\left(\Delta r_{1}^{\mathrm{II}}+\Delta r_{2}^{\mathrm{II}}+\Delta r_{1}^{\mathrm{IV}}+\Delta r_{2}^{\mathrm{IV}}\right) \\
& S_{3}=\frac{1}{\sqrt{2}}\left(\Delta r_{2}^{\mathrm{I}}+\Delta r_{1}^{\mathrm{III}}\right) \\
& S_{4}=\frac{1}{\sqrt{2}}\left(\Delta r_{1}^{\mathrm{I}}+\Delta r_{2}^{\mathrm{III}}\right) \\
& S_{5}=\frac{1}{\sqrt{2}}\left(\Delta \Theta^{\mathrm{I}}+\Delta \Theta^{\mathrm{III}}\right) \\
& S_{6}=\frac{1}{\sqrt{2}}\left(\Delta \Theta^{\mathrm{II}}+\Delta \Theta^{\mathrm{IV}}\right) \\
& S_{7}=\frac{1}{\sqrt{2}}\left(\Delta \theta^{\mathrm{I}}+\Delta \theta^{\mathrm{III}}\right) \\
& S_{8}=\frac{1}{\sqrt{2}}\left(\Delta \theta^{\mathrm{II}}+\Delta \theta^{\mathrm{IV}}\right) \\
& S_{9}=\frac{1}{\sqrt{8}}\left(\Delta \phi_{1}^{\mathrm{II}}+\Delta \phi_{2}^{\mathrm{II}}+\Delta \phi_{3}^{\mathrm{II}}+\Delta \phi_{4}^{\mathrm{II}}+\Delta \phi_{1}^{\mathrm{IV}}\right. \\
& \left.+\Delta \phi_{2}^{\mathrm{IV}}+\Delta \phi_{3}^{\mathrm{IV}}+\Delta \phi_{4}^{\mathrm{IV}}\right) \\
& S_{10}=\frac{1}{\sqrt{8}}\left(\Delta \phi_{1}^{\mathrm{II}}-\Delta \phi_{2}^{\mathrm{II}}-\Delta \phi_{3}^{\mathrm{II}}+\Delta \phi_{4}^{\mathrm{II}}-\Delta \phi_{1}^{\mathrm{IV}}\right. \\
& \left.+\Delta \phi_{2}^{\mathrm{IV}}+\Delta \phi_{3}^{\mathrm{IV}}-\Delta \phi_{4}^{\mathrm{IV}}\right) \\
& S_{11}=\frac{1}{2}\left(\Delta \phi_{2}^{\mathrm{I}}+\Delta \phi_{4}^{\mathrm{I}}+\Delta \phi_{1}^{\mathrm{III}}+\Delta \phi_{3}^{\mathrm{III}}\right) \\
& S_{12}=\frac{1}{2}\left(\Delta \phi_{1}^{\mathrm{I}}+\Delta \phi_{3}^{\mathrm{I}}+\Delta \phi_{2}^{\mathrm{III}}+\Delta \phi_{4}^{\mathrm{III}}\right) \\
& a_{2} \text { coordinates } \\
& S_{\mathrm{I}}=\frac{1}{2}\left(\Delta R^{\mathrm{I}}+\Delta R^{\mathrm{II}}-\Delta R^{\mathrm{III}}-\Delta R^{\mathrm{IV}}\right) \\
& S_{2}=\frac{1}{2}\left(\Delta r_{1}^{\mathrm{II}}+\Delta r_{2}^{\mathrm{II}}-\Delta r_{1}^{\mathrm{IV}}-\Delta r_{2}^{\mathrm{IV}}\right) \\
& S_{3}=\frac{1}{\sqrt{2}}\left(\Delta \Theta^{\mathrm{II}} \mathrm{I}-\Delta \Theta^{\mathrm{IV}}\right) \\
& S_{4}=\frac{1}{\sqrt{2}}\left(\Delta \theta^{\mathrm{II}}-\Delta \theta^{\mathrm{IV}}\right) \\
& S_{5}=\frac{1}{\sqrt{8}}\left(\Delta \phi_{1}^{\mathrm{II}}+\Delta \phi_{2}^{\mathrm{II}}+\Delta \phi_{3}^{\mathrm{II}}+\Delta \phi_{4}^{\mathrm{II}}-\Delta \phi_{1}^{\mathrm{IV}}\right. \\
& \left.-\Delta \phi_{2}^{\mathrm{IV}}-\Delta \phi_{3}^{\mathrm{IV}}-\Delta \phi_{4}^{\mathrm{IV}}\right) \\
& S_{6}=\frac{1}{\sqrt{8}}\left(\Delta \phi_{1}^{\mathrm{II}}-\Delta \phi_{2}^{\mathrm{II}}-\Delta \phi_{3}^{\mathrm{II}}+\Delta \phi_{4}^{\mathrm{II}}+\Delta \phi_{1}^{\mathrm{IV}}\right. \\
& \left.-\Delta \phi_{2}^{\mathrm{IV}}-\Delta \phi_{3}^{\mathrm{IV}}+\Delta \phi_{4}^{\mathrm{IV}}\right) \\
& S_{7}=\frac{1}{2}\left(\Delta \phi_{2}^{\mathrm{I}}-\Delta \phi_{4}^{\mathrm{I}}-\Delta \phi_{1}^{\mathrm{III}}+\Delta \phi_{3}^{\mathrm{III}}\right) \\
& S_{8}=\frac{1}{2}\left(\Delta \phi_{1}^{\mathrm{I}}-\Delta \phi_{3}^{\mathrm{I}}-\Delta \phi_{2}^{\mathrm{III}}+\Delta \phi_{4}^{\mathrm{III}}\right) \\
& b_{1} \text { coordinates } \\
& S_{1}=\frac{1}{2}\left(\Delta R^{\mathrm{I}}-\Delta R^{\mathrm{II}}-\Delta R^{\mathrm{III}}+\Delta R^{\mathrm{IV}}\right) \\
& S_{2}=\frac{1}{2}\left(\Delta r_{1}^{\mathrm{II}}-\Delta r_{2}^{\mathrm{II}}-\Delta r_{1}^{\mathrm{IV}}-\Delta r_{2}^{\mathrm{IV}}\right) \\
& S_{2}=\frac{1}{\sqrt{2}}\left(\Delta r_{2}^{\mathrm{I}}-\Delta r_{1}^{\mathrm{III}}\right) \\
& S_{4}=\frac{1}{\sqrt{2}}\left(\Delta r_{1}^{\mathrm{I}}-\Delta r_{2}^{\mathrm{III}}\right) \\
& S_{5}=\frac{1}{\sqrt{2}}\left(\Delta \Theta^{\mathrm{I}}-\Delta \Theta^{\mathrm{III}}\right) \\
& S_{6}=\frac{1}{\sqrt{2}}\left(\Delta \theta^{\mathrm{I}}-\Delta \theta^{\mathrm{III}}\right) \\
& S_{7}=\frac{1}{\sqrt{8}}\left(\Delta \phi_{1}^{\mathrm{II}}+\Delta \phi_{2}^{\mathrm{II}}-\Delta \phi_{3}^{\mathrm{II}}-\Delta \phi_{4}^{\mathrm{II}}-\Delta \phi_{1}^{\mathrm{IV}}\right. \\
& \left.-\Delta \phi_{2}^{\mathrm{IV}}+\Delta \phi_{3}^{\mathrm{IV}}+\Delta \phi_{4}^{\mathrm{IV}}\right) \\
& S_{8}=\frac{1}{\sqrt{8}}\left(\Delta \phi_{1}^{\mathrm{II}}-\Delta \phi_{2}^{\mathrm{II}}+\Delta \phi_{3}^{\mathrm{II}}-\Delta \phi_{4}^{\mathrm{II}}+\Delta \phi_{1}^{\mathrm{IV}}\right. \\
& \left.-\Delta \phi_{2}^{\mathrm{IV}}+\Delta \phi_{3}^{\mathrm{IV}}-\Delta \phi_{4}^{\mathrm{IV}}\right) \\
& S_{9}=\frac{1}{2}\left(\Delta \phi_{2}^{\mathrm{I}}+\Delta \phi_{4}^{\mathrm{I}}-\Delta \phi_{1}^{\mathrm{III}}-\Delta \phi_{3}^{\mathrm{III}}\right) \\
& S_{10}=\frac{1}{2}\left(\Delta \phi_{1}^{\mathrm{I}}+\Delta \phi_{3}^{\mathrm{I}}-\Delta \phi_{2}^{\mathrm{III}}-\Delta \phi_{4}^{\mathrm{III}}\right) \\
& S_{1}=\frac{1}{2}\left(\Delta R^{\mathrm{I}}-\Delta R^{\mathrm{II}}+\Delta R^{\mathrm{III}}-\Delta R^{\mathrm{IV}}\right) \\
& S_{2}+\frac{1}{2}\left(\Delta r_{1}^{\mathrm{II}}-\Delta r_{2}^{\mathrm{II}}-\Delta r_{1}^{\mathrm{IV}}+\Delta r_{2}^{\mathrm{IV}}\right) \\
& S_{3}=\frac{1}{\sqrt{8}}\left(\Delta \phi_{1}^{\mathrm{II}}+\Delta \phi_{2}^{\mathrm{II}}-\Delta \phi_{3}^{\mathrm{II}}-\Delta \phi_{4}^{\mathrm{II}}+\Delta \phi_{1}^{\mathrm{IV}}\right. \\
& \left.+\Delta \phi_{2}^{\mathrm{IV}}-\Delta \phi_{3}^{\mathrm{IV}}-\Delta \phi_{4}^{\mathrm{IV}}\right) \\
& S_{4}=\frac{1}{\sqrt{8}}\left(\Delta \phi_{1}^{\mathrm{II}}-\Delta \phi_{2}^{\mathrm{II}}+\Delta \phi_{3}^{\mathrm{II}}-\Delta \phi_{4}^{\mathrm{II}}-\Delta \phi_{1}^{\mathrm{IV}}\right. \\
& \left.+\Delta \phi_{2}^{\mathrm{IV}}-\Delta \phi_{3}^{\mathrm{IV}}+\Delta \phi_{4}^{\mathrm{IV}}\right) \\
& S_{5}=\frac{1}{2}\left(\Delta \phi_{2}^{\mathrm{I}}-\Delta \phi_{4}^{\mathrm{I}}+\Delta \phi_{1}^{\mathrm{III}}-\Delta \phi_{3}^{\mathrm{III}}\right) \\
& S_{6}=\frac{1}{2}\left(\Delta \phi_{1}^{\mathrm{I}}-\Delta \phi_{3}^{\mathrm{I}}+\Delta \phi_{2}^{\mathrm{III}}-\Delta \phi_{4}^{\mathrm{III}}\right)
\end{aligned}
$$


As can be seen from Table 7, however, the calculated frequencies of syndiotactic structure are not in agreement with the observed bands. Particularly the calculated $A_{2}$ modes do not fit the observed frequencies based on the vibrational selection rules. Zerbi [3] preferred the syndiotactic model to analyze the IR spectrum of PVF particularly because of the occurrence of the two C-F stretching modes (at 1144 and $1094 \mathrm{~cm}^{-1}$ ) with proper polarization. However, normal coordinate calculation suggests that the band at 1144 $\mathrm{cm}^{-1}$ is not a single mode but a mixed one. This suggestion is supported by normal coordinate calculations of 2-fluoropropane by Crowder [18] and 2-fluorobutane by us. In Table 3 for 2-fluorobutane, there are two modes strongly involving the stretching of the C-F bond (1142 and 892 $\mathrm{cm}^{-1}$ ). Moreover, it is not the high-frequency mode which contains the larger contribution from the $\mathrm{C}-\mathrm{F}$ stretching mode, though it is usually assumed as a representative of the C-F stretching in the empirical assignments. If we consider the observed band at $1144 \mathrm{~cm}^{-1}$ having a potential energy distribution, $\nu$ (CF) will be contributing mainly in the IR and $\gamma_{t}\left(\mathrm{CH}_{2}\right)$ in the Raman so that we can explain the behavior of the Raman 1150 and IR $1144 \mathrm{~cm}^{-1}$ bands in the observed spectra. In addition, the three infrared active species, $A_{1}, B_{1}$, and $B_{2}$ should have parallel dichroism when the molecular chains are oriented along the stretching direction. As shown in Table 7, however, the observed parallel bands do not correspond to calculated $B_{2}$ frequencies. They thus provide additional support for the atactic configuration in the crystal structure of PVF.

In order to have more conclusive evidence,

TABLE 7

Observed frequencies and calculated frequencies of syndiotactic PVF

\begin{tabular}{|c|c|c|c|c|c|c|}
\hline \multicolumn{2}{|l|}{ Observed } & \multicolumn{5}{|c|}{ Calculated } \\
\hline Infrared & Raman & $A_{1}$ & $A_{2}$ & $B_{1}$ & $B_{2}$ & Potential energy distribution \\
\hline & & 1460 & & & & 94(HH-b) \\
\hline \multicolumn{7}{|l|}{$1444 w^{a}$} \\
\hline $1426 \mathrm{~s}$ & $1436 \mathrm{~s}$ & & 1446 & & & 98(HH-b) \\
\hline $1410 \mathrm{~s}$ & & & & & 1407 & $37(\mathrm{HH}-\mathrm{w})$ \\
\hline $1367 \mathrm{~m}$ & $1366 \mathrm{~m}$ & & & & & \\
\hline $1354 \mathrm{~m}$ & $1354 \mathrm{~m}$ & & & & & \\
\hline \multirow[t]{2}{*}{$1300 \mathrm{vw}$} & $1302 \mathrm{~m}$ & & & & & \\
\hline & $1256 \mathrm{~m}$ & & & & & \\
\hline $1249 \mathrm{~m}$ & & & & 1247 & & 53(HH-w) 34(C-C s) 11(CH-pl) \\
\hline \multirow[t]{2}{*}{$1232 \mathrm{~m}$} & & & 1223 & & & $42(\mathrm{HH}-\mathrm{w}) 35(\mathrm{C}-\mathrm{C} \mathrm{s})$ \\
\hline & $1194 \mathrm{~m}$ & & & & & \\
\hline \multirow[t]{2}{*}{$1185 \mathrm{vw}$} & & & & & 1186 & 45(HH-w) 24(CH-pl) \\
\hline & $1150 \mathrm{~s}$ & & & & & \\
\hline $1144 \mathrm{~s}$ & & 1149 & & & & $61(\mathrm{HH}-\mathrm{tw}) 25(\mathrm{CX}-\mathrm{s})$ \\
\hline $1094 \mathrm{~s}$ & $1095 \mathrm{~m}$ & & & 1087 & & $56(\mathrm{CX}-\mathrm{s}) 22(\mathrm{HH}-\mathrm{r})$ \\
\hline $1084 \mathrm{~s}$ & & & 1062 & & & $84(\mathrm{HH}-\mathrm{tw})$ \\
\hline \multirow[t]{2}{*}{$1033 \mathrm{~s}$} & $1032 \mathrm{w}$ & 1011 & & & & $21(\mathrm{C}-\mathrm{C} \mathrm{s}) 19(\mathrm{CH}-\mathrm{pl}) 12(\mathrm{HH}-\mathrm{tw})$ \\
\hline & & & & & & $11(\mathrm{CX}-\mathrm{s})$ \\
\hline \multirow[t]{3}{*}{$966 \mathrm{vw}$} & $961 \mathrm{w}$ & & & & 960 & $39(\mathrm{CH}-\mathrm{pl}) 10(\mathrm{HH}-\mathrm{tw})$ \\
\hline & & & 950 & & & $52(\mathrm{CH}-\mathrm{pl}) 17(\mathrm{HH}-\mathrm{tw}) 11(\mathrm{C}-\mathrm{C} \mathrm{s})$ \\
\hline & & & & 944 & & $61(\mathrm{C}-\mathrm{C} s)$ \\
\hline $888 \mathrm{~m}$ & $890 \mathrm{~m}$ & 851 & & & & $37(\mathrm{CX}-\mathrm{s})$ \\
\hline $831 \mathrm{~s}$ & $834 s$ & & & 825 & & $81(H H-r)$ \\
\hline $815 w$ & & & & & 805 & $38(\mathrm{HH}-\mathrm{r}) 31(\mathrm{CX}-\mathrm{s}) 15(\mathrm{C}-\mathrm{C} \mathrm{s})$ \\
\hline $765 w^{a}$ & & & & & & \\
\hline $722 w^{a}$ & & & & & & \\
\hline
\end{tabular}

\footnotetext{
a Band due to head-to-head, tail-to-tail defect structure.
} 


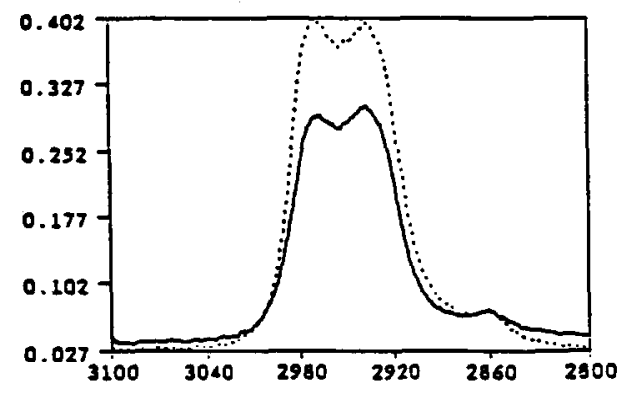

We turn now to an approximate normal coordinate analysis of the spectra of PVF based on the atactic model of the chain configuration.

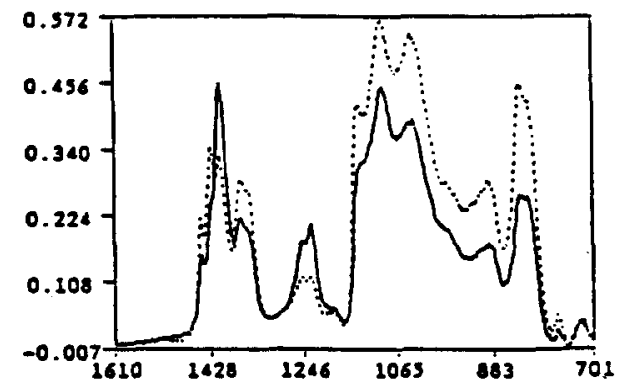

Fig. 4. Infrared spectra of an oriented sample of PVF in polarized light. - electric vector parallel to drawing direction; $\cdots \cdots$ electric vector perpendicular to drawing direction.

however, we need a more precise force field from additional model compounds like di- and eso2,4-difluoropentane, and 2,4,6-trifluoroheptane.

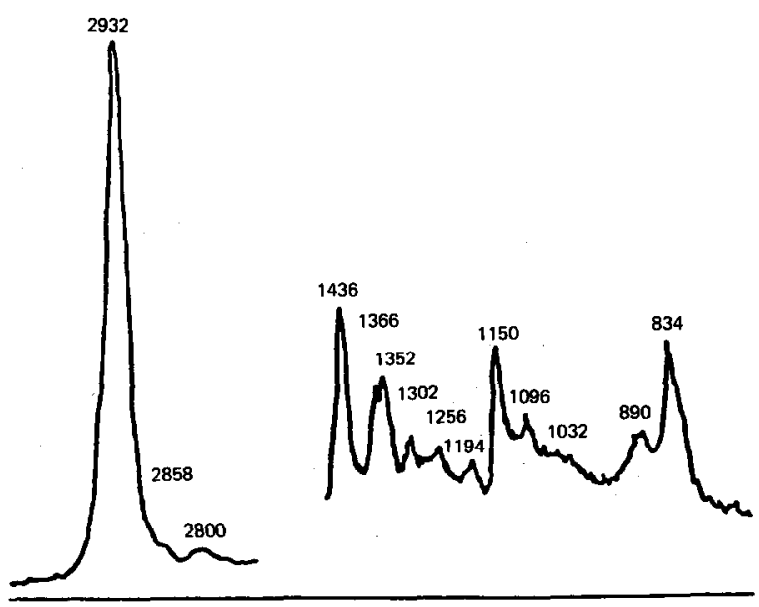

Fig. 5. Raman spectrum of poly(vinyl fluoride).

\section{ASSIGNMENTS OF BANDS IN THE SPECTRUM OF POLY(VINYL FLUORIDE)}

Before discussing the assignments of the bands in the infrared spectrum of PVF it is appropriate that we consider what frequencies may be expected to appear. For molecules with any appreciable symmetry this can be done by means of a group theoretical approach. In the present case the uncertainty in structure occasioned by the random location of the fluorine atoms does not make the group theory analysis particularly useful. We will therefore begin by classifying the normal modes of the molecule in terms of the separable modes of component groups in the chain. Later it will become more evident to what extent we may consider the modes to be separable and what influence neighboring chain interactions can be expected to have on the spectrum.

It seems reasonable to choose the monomer unit $-\mathrm{CH}_{2}-\mathrm{CHF}$ - as the fundamental spectroscopic unit, since, although two types of such units occur (depending on the position of the F), these are essentially independent of each other as a result of their random location in the chain. We therefore do not expect that interactions between these two types of units will give rise to any new frequencies in the spectrum.

With this assumption we expect $3 \times 6-4=14$ normal modes of the $-\mathrm{CH}_{2} \mathrm{CHF}-$ group in the polymer chain. In terms of separable modes of the component groups, the frequencies will be distributed as follows: $6 \mathrm{CH}_{2}$ (2 stretching, bending, wagging, twisting, rocking), $3 \mathrm{CH}, 3 \mathrm{CF}$ (each consisting of a stretching plus 2 bending modes, one perpendicular to the chain axis and one parallel), and $2 \mathrm{CC}$ (skeletal vibrations).

The vibrational assignment of PVF in terms of the atactic model is attempted below. The vibrational analysis is based on the infrared polarization and Raman data, and on the comparison with the normal coordinate analysis data of model compounds 2-fluoropropane and 2-fluorobutane. 
For the head-to-head defect structure, the infrared data [7] of isoregic PVF is used.

(1) Carbon-hydrogen stretching modes. Three bands are expected for the atactic structure: $\nu(\mathrm{CH}), \nu_{\mathrm{a}}\left(\mathrm{CH}_{2}\right)$, and $\nu_{\mathrm{s}}\left(\mathrm{CH}_{2}\right)$ which are the typical localized modes. The asymmetric and the symmetric $v\left(\mathrm{CH}_{2}\right)$ can be assigned to the perpendicular bands at 2969 and $2932 \mathrm{~cm}^{-1}$, respectively, which have the proper polarization and frequency. However, an explanation of the disappearance of the band at $2969 \mathrm{~cm}^{-1}$ in the Raman spectrum is puzzling because the asymmetric $\mathrm{CH}_{2}$ stretching vibration should be also Raman active due to its change of polarizability ellipsoid during the vibration. The assignment of the $\nu(\mathrm{CH})$ is difficult due to its low intensity as occurs in other vinyl polymers. The band at $2861 \mathrm{~cm}^{-1}$ is due to the isolated cthylcnc molecules in the tail-to-tail portion of polymer. This assignment is based on (a) the absence of such a band in the spectrum of isoregic PVF and (b) the presence of a similar band in polyvinylidene fluoride [25].

(2) $\mathrm{CH}_{2}$ deformation modes. Examination of potential energy distribution in the normal coordinate calculation for 2-fluorobutane suggests that only bending $\left[\delta\left(\mathrm{CH}_{2}\right)\right]$ and rocking $\left[\gamma_{\mathrm{r}}\left(\mathrm{CH}_{2}\right)\right]$ modes can be regarded as localized deformation modes (Table 3 ).

The assignment of the $\delta\left(\mathrm{CH}_{2}\right)$ to the 1426 $\mathrm{cm}^{-1}$ is based on the proper polarization (perpendicular) and presence of such a mode in the frequency range of the model compound (2-fluorobutane). The very high dichroic ratio and disappearance of this band in the molten state suggests its origin to an ordered structure with a localized mode. Normal coordinate analysis of syndiotactic PVF shows no coupling of this mode so a localized mode can be accepted.

The strong perpendicular band at $831 \mathrm{~cm}^{-1}$ can be undoubtedly assigned as a $\gamma_{\mathrm{r}}\left(\mathrm{CH}_{2}\right)$ mode. Normal coordinate analysis of 2-fluorobutane and syndiotactic PVF supports this assignment as a localized mode.

The bands at 1444 and $760 \mathrm{~cm}^{-1}$ are due to the tail-to-tail structure because of their absence in the spectrum of isoregic PVF. Although normal-mode analysis of model compounds such as 2,5-difluoroheptane is required, these bands can be assigned with some confidence as $\delta\left(\mathrm{CH}_{2} \mathrm{CH}_{2}\right)$ and $\gamma_{\mathrm{r}}\left(\mathrm{CH}_{2} \mathrm{CH}_{2}\right)$, respectively. The fact that its polarization properties are the same as those of the two neighboring localized bands $\left[\delta\left(\mathrm{CH}_{2}\right)\right.$ and $\gamma_{\mathrm{r}}\left(\mathrm{CH}_{2}\right)$, respectively], supports these assignments.

The assignment of the remaining deformation modes is difficult due to extensive coupling. Infrared polarization, the intensities of the infrared and Raman spectra, and the general frequency positions from normal coordinate analysis of model compound and syndiotactic PVF are helpful in assigning bands.

The band at $1410 \mathrm{~cm}^{-1}$ shows a relatively high parallel dichroism indicating that it is predominantly a $\gamma_{\mathrm{w}}\left(\mathrm{CH}_{2}\right)$ mode. However, the disappearance of this band in the Raman spectrum is unexpected because carbon-fluorine stretching modes are not in this region.

We have a reasonable suggestion for the location of the $\gamma_{\mathrm{t}}\left(\mathrm{CH}_{2}\right)$ mode. Although this mode is predicted to be very weak in the infrared spectrum, the normal coordinate analysis of 2-fluorobutane and syndiotactic PVF and Raman data suggest that this mode contributes predominantly to the Raman band at $1150 \mathrm{~cm}^{-1}$. As discussed, this mode is strongly mixed with the $\nu$ (CF) mode. The Raman band at $1302 \mathrm{~cm}^{-1}$ can also be assigned to the $\gamma_{\mathrm{t}}\left(\mathrm{CH}_{2}\right)$ mode which is absent from the infrared spectra.

(3) Carbon-fluorine stretching modes. One of the most striking characteristics of infrared spectra of fluorinated organic structures is the strong intensity of the carbon-fluorine stretching vibrations. It is due to the fact that the stretching vibrations of highly polar linkages are associated with high infrared intensities due to the changes in the permanent dipole moment. The intensities of the Raman lines, which are governed by changes in the induced dipole moment during vibration, are in contrast low for these vibrations and are thus not a distinguishing feature of the spectra. A second important aspect of the infrared spectra of carbon-fluorine stretching vibrations is the possibility of coupling (notable with skeletal stretching), which prohibits its providing unique group frequencies and results in very complex absorption patterns. In spite of the 
above facts, we will attempt to make assignments for bands involving carbon-fluorine stretching modes based on the normal coordinate analysis of 2-fluoropropane [18] and 2-fluorobutane and on the relative intensities of infrared and Raman spectra.

In 2-fluoropropane, the $\nu$ (CF) modes lie within the range $1264-819 \mathrm{~cm}^{-1}$. According to our work on 2-fluorobutane, this mode falls in the 1142-892 $\mathrm{cm}^{-1}$ region, respectively. Therefore, the perpendicularly polarized bands at 1144,1033 , and 888 $\mathrm{cm}^{-1}$ can be assigned to the modes involving $\nu(\mathrm{CF})$. A comparison of the intensities in infrared and Raman spectra with the required polarization supports these assignments. For the band at $888 \mathrm{~cm}^{-1}$, it is believed to be coupled strongly with the $\nu(\mathrm{CC})$ mode. The fact of the strong intensity in infrared and the weak in the Raman band at $1033 \mathrm{~cm}^{-1}$ suggest that this band is predominantly the $\nu(C F)$ mode. As discussed, the band at $1144 \mathrm{~cm}^{-1}$ can be assigned to contributions from the $\gamma_{\mathrm{t}}\left(\mathrm{CH}_{2}\right)$ and $\nu(\mathrm{CF})$ modes.

(4) Carbon-fluorine deformation modes. There is little doubt that the two bands at 450 and 402 $\mathrm{cm}^{-1}$ can bc assigned to $\gamma_{\mathrm{r}}(\mathrm{CF})$ and $\gamma_{w}$ (CF) which have the proper polarization and frequency. Normal coordinate analyses of 2-fluorobutane and syndiotactic PVF support these assignments.

(5) Carbon-carbon stretching and carbon-hydrogen deformation modes. The assignment of the remaining bands is more difficult due to extensive coupling of the modes and the lack of data from deuterated polymers. The above mentioned studies on carbon-carbon frequencies of small molecules and syndiotactic PVF show that the $\nu(\mathrm{CC})$ modes occur in a broad frequency range; that is, these are delocalized modes. Therefore, it is hard to make unambiguous assignments of the $\nu(\mathrm{CC})$ modes of PVF. However, the fact that an intense Raman band arises from bonds having nearly symmetrical charge distributions such as $\mathrm{C}-\mathrm{C}$ can lead to a fairly reliable assignment of this mode. Although couplings are expected, the $\gamma_{w}(\mathrm{CH})$ and $\gamma_{r}(\mathrm{CH})$ modes occur in fairly restricted frequency ranges, around 1200 and $1300 \mathrm{~cm}^{-1}$. The group of bands at 1232 and $1249 \mathrm{~cm}^{-1}$ and at 1.354 and $1367 \mathrm{~cm}^{-1}$ are proba-
TABLE 8

Proposed vibrational assignment of PVF in terms of atactic configuration

\begin{tabular}{|c|c|c|c|}
\hline \multicolumn{2}{|l|}{ Observed } & \multirow{2}{*}{$\begin{array}{l}\text { Infrared } \\
\text { polarization }\end{array}$} & \multirow{2}{*}{$\begin{array}{l}\text { Approximate } \\
\text { assignment }\end{array}$} \\
\hline Infrared & Raman & & \\
\hline $2970 \mathrm{~s}$ & & $\perp$ & $\overline{\nu_{\mathrm{a}}\left(\mathrm{CH}_{2}\right)}$ \\
\hline $2932 \mathrm{~s}$ & $2932 \mathrm{~s}$ & $\perp$ & $\nu_{\mathrm{s}}\left(\mathrm{CH}_{2}\right)$ \\
\hline $2861 \mathrm{w}$ & $2859 \mathrm{w}$ & & $\nu\left(\mathrm{CH}_{2} \mathrm{CH}_{2}\right)$ \\
\hline $1446 \mathrm{w}$ & & $\perp$ & $\delta\left(\mathrm{CH}_{2} \mathrm{CH}_{2}\right)$ \\
\hline $1427 \mathrm{~s}$ & $1436 \mathrm{~s}$ & $\perp$ & $\delta\left(\mathrm{CH}_{2}\right)$ \\
\hline $1410 s$ & & $\|$ & $\gamma_{w}\left(\mathrm{CH}_{2}\right)$ \\
\hline $1368 \mathrm{~m}$ & $1366 \mathrm{~m}$ & $\perp$ & $\gamma_{\mathrm{r}}(\mathrm{CH})+\gamma_{\mathrm{w}}\left(\mathrm{CH}_{2}\right)$ \\
\hline $1351 \mathrm{~m}$ & $1354 \mathrm{~m}$ & $\perp$ & $\gamma_{\mathrm{r}}(\mathrm{CH})+\nu(\mathrm{CC})$ \\
\hline $1295 \mathrm{ww}$ & $1302 \mathrm{~m}$ & & $\gamma_{t}\left(\mathrm{CH}_{2}\right)$ \\
\hline $1250 \mathrm{~m}$ & $1256 \mathrm{~m}$ & $\|$ & $\gamma_{w}(\mathrm{CH})+\nu(\mathrm{CC})$ \\
\hline $1232 \mathrm{~m}$ & & $\|$ & $\gamma_{w}(\mathrm{CH} \mathrm{CH}),+\gamma_{w}(\mathrm{CH})$ \\
\hline $1189 \mathrm{vw}$ & $1194 \mathrm{~m}$ & & $\nu(\mathrm{CC})$ of $\mathrm{T}-\mathrm{T}$ \\
\hline $1144 \mathrm{~s}$ & $1150 \mathrm{~s}$ & $\perp$ & $\nu(\mathrm{CF}),+\gamma_{t}\left(\mathrm{CH}_{2}\right)$ \\
\hline $1092 \mathrm{~s}$ & $1095 \mathrm{~m}$ & $\perp$ & $\nu(\mathrm{CC})+\nu(\mathrm{CF})$ \\
\hline $1033 \mathrm{~s}$ & $1032 \mathrm{w}$ & $\perp$ & $\nu(\mathrm{CF})$ \\
\hline $965 \mathrm{vw}$ & $961 \mathrm{vw}$ & $\perp$ & \\
\hline $888 \mathrm{~m}$ & $890 \mathrm{~m}$ & $\perp$ & $\nu(\mathrm{CF})+\nu(\mathrm{CC})$ \\
\hline $831 \mathrm{~s}$ & $834 \mathrm{~s}$ & $\perp$ & $\gamma_{\mathrm{r}}\left(\mathrm{CH}_{2}\right)$ \\
\hline $763 w$ & & $\perp$ & $\gamma_{\mathbf{r}}\left(\mathrm{CH}_{2} \mathrm{CH}_{2}\right)$ \\
\hline $722 w$ & $720 \mathrm{vw}$ & $\|$ & \\
\hline $510 \mathrm{vw}$ & $506 w$ & $\|$ & \\
\hline $465 \mathrm{~m}$ & $454 \mathrm{~m}$ & $\perp$ & $\gamma_{\mathrm{r}}(\mathrm{CF})$ \\
\hline $394 \mathrm{~m}$ & $395 w$ & $\|$ & $\gamma_{w}(C F)$ \\
\hline
\end{tabular}

bly assignable to the modes involved in $\gamma_{\mathrm{w}}(\mathrm{CH})$ and $\gamma_{\mathrm{r}}(\mathrm{CH})$, respectively. The strong parallel polarization of these former two bands and the strong perpendicular polarization of the latter two bands support these assignments. Comparison of the Raman and infrared intensities and the normal-mode analyses suggest that the band at $1249 \mathrm{~cm}^{-1}$ is the coupled $\gamma_{\mathrm{w}}(\mathrm{CH})$ and $\nu(\mathrm{CC})$ mode, the band at $1232 \mathrm{~cm}^{-1}$ being the uncoupled $\gamma_{w}(\mathrm{CH})$ mode. It is also suggested that the $1367 \mathrm{~cm}^{-1}$ is the coupled $\gamma_{\mathrm{r}}(\mathrm{CH})$ and $\gamma_{\mathrm{w}}\left(\mathrm{CH}_{2}\right)$ mode, and the $1354 \mathrm{~cm}^{-1}$ band the coupled $\gamma_{\mathrm{r}}(\mathrm{CH})$ and $\nu(\mathrm{CC})$ mode.

Although uncertainties will always exist, the overall set of proposed assignments for PVF is quite satisfactory. The assignments proposed tentatively are in Table 8 . Several assignments require additional confirmation which could be achieved by studies of deuterated PVF and extended studies on low-molecular-weight model compounds. 


\section{Conclusions}

We have developed a force field for secondary fluorides based on the extension of existing force fields for hydrocarbons and primary fluorides. We recognize that this force field cannot be regarded as a general force field for secondary fluorides. However, the agreement between the observed and calculated frequencies below 1200 $\mathrm{cm}^{-1}$ indicates that it is presently useful to apply to the analysis of the spectra of poly(vinyl fluoride).

A comparison of observed infrared and Raman bands with frequencies calculated for syndiotactic PVF shows that PVF produced by conventional free radical polymerization has an atactic structure, supporting ${ }^{19}$ F-NMR results and conclusions reached by Koenig and Boerio.

Band assignments in terms of atactic structure are also proposed.

The authors acknowledge the support of this research by E.I. duPont De Nemours Company and the Edison Polymer Innovation Corporation (EPIC).

\section{REFERENCES}

1 J.L. Koenig and J.J. Mannion, J. Polym. Sci. Part A2, 4 (1966) 401.

2 J.L. Koenig and F.J. Boerio, Makromol. Chemie, 125 (1969) 302.

3 G. Zerbi and G. Cortelli, Spectrochim. Acta, 26 (1970) 733.

4 G. Natta, I.W. Bassi and G. Allegra, Atti. Accad. Naz. Lincei Cl. Sci. Fis. Mat. Nat. Rend., 31 (1961) 350.
5 M.D. Bruch, F.A. Bovey and R.E. Cais, Macromolecules, 17 (1984) 2547.

6 R.E. Cais and J.M. Kometani, in J.C. Randall (Ed.), NMR and Macromolecules, Am. Chem. Soc. Symp. Ser., 247 (1984) 153.

7 J.W. Hong, Ph.D. Thesis, Case Western Reserve University, Cleveland, $\mathrm{OH}, 1991$.

8 T. Shimanouchi, J. Polym. Sci. Part C, 7 (1965) 85.

9 A. Abragam, Principles of Nuclear Magnetism, Oxford University Press, Oxford, 1986.

10 E. Benedetti and P. Cecchi, Spectrochim. Acta, Part A, 28 (1972) 1007.

11 S. Mizushima, T. Shimanouchi, K. Nakamura, M. Hayashi and S. Tsuchiya, J. Chem. Phys., 26 (1957) 970.

12 J.J. Shipman, V.L. Folt and S. Krimm, Spectrochim. Acta, 18 (1962) 1603.

13 G.G. Opaskar and S. Krimm, Spectrochim. Acta Part A, 23 (1967) 2261.

14 P.N. Gates, E.F. Mooney and H.A. Willis, Spectrochim. Acta, Part A, 23 (1967) 2043.

15 W.H. Moore, Ph. D. Thesis, University of Michigan, 1972.

16 X. Jing and S. Krimm, Spectrochim. Acta, Part A, 39 (1983) 251.

17 G.A. Crowder and T. Koger, J. Mol. Struct., 29 (1975) 233.

18 G.A. Crowder and T. Koger, J. Mol. Struct., 23 (1974) 311.

19 J.H. Griffiths, N.L. Owen and J. Sheridan, J. Chem. Soc. Faraday Trans., 11 (1973) 1359.

20 G.A. Crowder and H.K. Mao, J. Mol. Struct., 18(1) (1973) 33.

21 G.A. Crowder and H.K. Mao, J. Mol. Struct., 23 (1974) 161.

22 R.G. Snyder and J.H. Dennison, J. Chem. Phys., 20 (1952) 313.

23 J.H. Schachtschneider and R.G. Snyder, Spectrochimica Acta, 19 (1963) 117.

24 P.C. Painter, M.M. Coleman and J.L. Koenig, The Theory of Vibrational Spectroscopy and its Application to Polymeric Materials, Wiley, New York, 1982, Chap. 4.

25 J.F. White, J. Polym. Sci. Part A, 3 (1965) 309. 